\title{
Research on Self-Calibration of HF Ground Wave Radar
}

\author{
Antenna Arrays \\ Wu Rui, Wen Bi-yang, Zhong Zhi-feng, Hong Chun \\ (School of Electronic Information, Wuhan University, Wuhan, Hubei 430079, China)
}

Abstract: Since the performance of high-resolution direction finding algorithm for HF Ground Wave Radar (GWR) is severely degraded by sensor phase and amplitude errors, the radar system's phase calibration is the prerequisite of keeping the radar working in order. According to the characteristic of HF GWR's sea echo, this paper, based on an arbitrary triangular array, presents that Space -Time DOA(direction of arrival) Matrix Method, which is used to estimate 2D DOA under ideal conditions, can be used to estimate planar wave's DOA and sensor phase and amplitude errors simultaneously so as to achieve self-calibration. Its validity is verified not only by computer simulation, but also by comparing treatment results of measured data before and after calibration with the GPS-measured result.

Key words: HF Ground Wave Radar, Space-Time DOA Matrix Method, Triangular Array, Self-Calibration

This project is supported by National 863 Program (No.2001AA631050).

\section{Introduction}

In fact, most of the recent high-resolution spatial spectrum estimation algorithms (such as MUSIC $^{[1]}$, ESPRIT $^{[2]}$, et al), which perform well, are based on the assumption that the array manifold is known exactly. However, in a practical system, these algorithms are severely degraded and even out of work ${ }^{[3,4,5]}$ due to unavoidable perturbations in array manifold. Calibration with auxiliary sources and self-calibration are the two normal ways of array calibration. Calibration with auxiliary sources is impractical because it requires the exact position information of the sources and costs very much. Self-calibration is the nowadays research focus, and many self-calibration algorithms have arose.

An HF Ground Wave Radar, working in shortwave band, usually consists of a large phase-controlled antenna array close to the seashore. It's difficult to calibrate this system's antenna array with auxiliary sources. So the problem becomes significant that how to calibrate the radar system' channels by making use of detected targets. As to HF GWR, it is unnecessary to estimate the elevation of the targets' echo that comes mainly from ocean's surface, so the HF Ground wave radar's antenna system is usually a uniform linear array (ULA). The two-dimension array constructed by adding new antennae to the ULA can estimate the 2D direction of arrival (DOA) of targets' echo. The two-dimension array will get redundant information when the DOA of echo is just one dimensional. Based on the premise that echo is one dimensional, our primary idea is to exploit the redundancy in order to achieve the HF Ground wave radar's channels' self-calibration.

In this paper, the shape of the array consisting of three whips is an arbitrary triangle. Space -Time DOA Matrix Method was initially devised to estimate 2D DOA based on precise knowledge of array manifold, but its formula under amplitude and phase errors can be deduced when echo is planar wave. DOA and phase perturbations can be estimated simultaneously through the redundancy generated by the 2D DOA technique applied to one dimension DOA estimation.

\section{2、 Signal Model}

Consider a triangular array with 3 sensors $\left(X_{1}, X_{2}, X_{M}\right)$ as Fig.1 shows. $X_{1}$ lies on the grid 
origin and $X_{2}$ lies on the $\mathrm{X}$-axis whose distance from $X_{1}$ is $d_{x} \cdot X_{M}$, an arbitrary point on the XY-plane, is $d_{y}$ away from $\mathrm{X}$-axis and $r$ from the origin. Setting $X_{1}$ as the norm, each sensor's amplitude and phase error relative to $X_{1}$ can be written

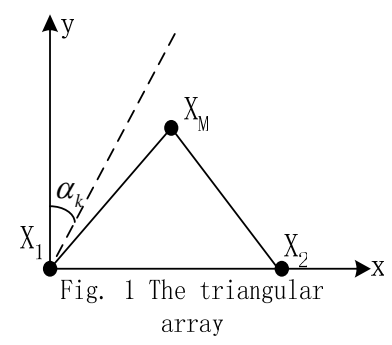
as $G_{1} \exp \left(j \varphi_{1}\right), G_{2} \exp \left(j \varphi_{2}\right)$ and $G_{M} \exp \left(j \varphi_{M}\right)$. Obviously, it is easy to get $G_{1}=1$ and $\varphi_{1}=0$ from the definition. Assume that there are $d$ narrowband and mutually uncorrelated sources entered at wavelength $\lambda$ and sufficiently far from the array to allow a planar wavefront. Additive noise is present at each sensor and is assumed to be zero mean, Gaussian process. The received signal of each sensor $x_{i}(t)$ is given by

$$
\begin{aligned}
& x_{i}(t)=G_{i} \sum_{k=1}^{d} s_{k}(t) \cdot \exp \left[j \frac{2 \pi}{\lambda}(i-1) d_{x} \sin \alpha_{k}\right] \cdot \exp \left[j \varphi_{i}\right]+n_{i}(t), \quad(i=1,2) \\
& x_{M}(t)=G_{M} \sum_{k=1}^{d} s_{k}(t) \cdot \exp \left[j \frac{2 \pi}{\lambda} d_{p}\right] \cdot \exp \left[j \varphi_{M}\right]+n_{M}(t)
\end{aligned}
$$

where $s_{k}(t)$ denotes the signal emitted by the $k$-th source, $\alpha_{k}$ denotes the direction of arrival of the $k$-th source, $n_{i}(t)$ is additive noise with variance $\sigma_{i}^{2}$ and independent from sensor to sensor and $d_{p}=d_{y} \cos \alpha_{k}+\sqrt{r^{2}-d_{y}^{2}} \sin \alpha_{k}$.

\section{3、 Extended Spatial Time DOA Matrix Method}

Original Space-Time DOA Matrix Method was devised to estimate 2D DOA of received signals under ideal conditions when $X_{M}$ lies on Y-axis. Based on the signal Model described in section 2, Space-Time DOA Matrix Method can be rewritten as follows:

(1), (2) are written in a matrix form as:

$$
\begin{aligned}
& X(t)=A s(t)+n(t) \\
& X=\left[x_{1}(t), x_{2}(t), x_{M}(t)\right]^{T}, n=\left[n_{1}(t), n_{2}(t), n_{M}(t)\right]^{T}, s=\left[s_{1}(t), \cdots, s_{d}(t)\right]^{T} \\
& A=\left[\mathbf{a}_{1}, \cdots, \mathbf{a}_{d}\right], \mathbf{a}_{k}=\left[a_{1 k}, a_{2 k}, a_{M k}\right] \\
& a_{i k}=G_{i} \exp \left[j \frac{2 \pi}{\lambda}(i-1) d_{x} \sin \alpha_{k}+j \varphi_{i}\right], \quad(i=1,2) \\
& a_{M k}=G_{M} \exp \left[j \frac{2 \pi}{\lambda} d_{p}+j \varphi_{M}\right]
\end{aligned}
$$


According to the definition of correlation, the correlation matrices of $x_{M}(t)$ and $x_{2}(t)$ with each sensor are

$$
\begin{aligned}
R_{x_{i} x_{M}}(\tau) & =E\left\{x_{i}\left(t+\frac{\tau}{2}\right) x_{M}^{*}\left(t-\frac{\tau}{2}\right)\right\} \\
& =\sum_{k=1}^{d}\left[R_{s_{k} s_{k}}(\tau) a_{M k}^{*}\right] \cdot a_{i k},(\tau \neq 0, i=1,2,3) \\
R_{x_{i} x_{2}}(\tau) & =E\left\{x_{i}\left(t+\frac{\tau}{2}\right) x_{2}^{*}\left(t-\frac{\tau}{2}\right)\right\} \\
& =\sum_{k=1}^{d}\left[R_{s_{k} s_{k}}(\tau) a_{2 k}^{*}\right] \cdot a_{i k} \\
& =\sum_{k=1}^{d}\left[R_{s_{k} s_{k}}(\tau) a_{M k}^{*}\right] \cdot a_{i k} \cdot \frac{a_{2 k}^{*}}{a_{M k}^{*}},(\tau \neq 0, i=1,2,3)
\end{aligned}
$$

Where $R_{X}(\tau)=\left[R_{x_{1} X_{M}}(\tau), R_{x_{2} x_{M}}(\tau), R_{x_{M} X_{M}}(\tau)\right]^{T}, \quad R_{Y}(\tau)=\left[R_{x_{1} x_{2}}(\tau), R_{x_{2} x_{2}}(\tau), R_{x_{M} x_{2}}(\tau)\right]^{T}$, $R_{S}(\tau)=\left[R_{s_{1} S_{1}}(\tau) \cdot a_{M 1}^{*}, R_{s_{1} S_{1}}(\tau) \cdot a_{M 2}^{*}, \cdots, R_{s_{d} s_{d}}(\tau) \cdot a_{M d}^{*}\right]^{T}$.Thus (8) and (9) can be rewritten as

$$
R_{X}(\tau)=A R_{S}(\tau), R_{Y}(\tau)=A \Phi R_{S}(\tau)
$$

where $\Phi$ is $D \times D$ dimensional diagonal matrix.

$$
\begin{aligned}
\Phi & =\operatorname{diag}\left[\frac{a_{21}^{*}}{a_{M 1}^{*}}, \cdots \frac{a_{2 k}^{*}}{a_{M k}^{*}}, \cdots \frac{a_{2 d}^{*}}{a_{M d}^{*}}\right] \\
& =\frac{G_{i}}{G_{M}} \operatorname{diag}\left[e^{j \frac{2 \pi}{\lambda}\left(d_{y} \cos \alpha_{1}-d_{a} \sin \alpha_{1}+\varphi_{M}-\varphi_{2}\right)}, \cdots, e^{j \frac{2 \pi}{\lambda}\left(d_{y} \cos \alpha_{1}-d_{a} \sin \alpha_{1}+\varphi_{M}-\varphi_{2}\right)}\right]
\end{aligned}
$$

Respectively, when $\tau=T_{s}, 2 T_{s}, \cdots N T_{s}$, we can get $\mathrm{N}$ groups of $R_{X}(\tau), R_{Y}(\tau)$ and construct the following matrices.

$$
\begin{gathered}
X=\left[R_{X}\left(T_{s}\right), R_{X}\left(2 T_{s}\right), \cdots, R_{X}\left(N T_{s}\right)\right] \\
Y=\left[R_{Y}\left(T_{s}\right), R_{Y}\left(2 T_{s}\right), \cdots, R_{Y}\left(N T_{s}\right)\right]
\end{gathered}
$$

From (10), we can get

$$
X=A S, \quad Y=A \Phi S
$$

where $S=\left[R_{S}\left(T_{s}\right), R_{S}\left(2 T_{s}\right), \cdots, R_{S}\left(N T_{s}\right)\right]$.

The spatial time DOA matrix is constructed as $R_{T S}=Y \cdot[X]^{-} \quad$ (15), where $[X]$ denotes the pseudo inverse $[X]^{-}=X^{H}\left(X X^{H}\right)^{-1}$.

It is known from [9] that if both A and B are full rank, if all the diagonal elements of $\Phi$ are 
different, D nonzero eigenvalues of $R_{T S}$ are equal to D diagonal elements of $\Phi$ and moreover these eigenvalues' corresponding eigenvectors are equal to the corresponding signal steering vectors.

$$
R_{T S} A=A \Phi
$$

So we eigen-decompose $R_{T S}$ to obtain A and $\Phi$. For A is $3 \times d$ dimensional, the biggest

number of DOA that can be estimated is 2 . Through $k$-th $(0<k<3)$ source's steering vector $\mathbf{a}_{k}$,

it is easy to get it is easy to get $a_{2 k}=G_{2} e^{j \frac{2 \pi}{\lambda} d_{x} \sin \alpha_{k}+j \varphi_{2}}$ and $a_{M k}=G_{M} e^{j \frac{2 \pi}{\lambda} d_{p}+j \varphi_{M}}$ whose

corresponding eigenvalue is $\Phi(k, k)=\frac{G_{2}}{G_{M}} e^{j \frac{2 \pi}{\lambda}\left(d_{y} \cos \alpha_{k}-d_{a} \sin \alpha_{k}+\varphi_{M}-\varphi_{2}\right)}$.

\section{The algorithm}

In summary, the steps of the algorithm are:

Step1: estimate the number of sources in the received signal;

Step2: construct spatial time DOA matrix $R_{T S}$ from (8), (9), (12), (13);

Step3: eigen-decompose $R_{T S}$;

As to the $k$-th source, we can get 3 equations:

$$
\begin{gathered}
a_{2 k}=e^{j \frac{2 \pi}{\lambda} d_{x} \sin \alpha_{k}+j \varphi_{2}} \\
a_{M k}=e^{j \frac{2 \pi}{\lambda} d_{y} \cos \alpha_{k}+j \frac{2 \pi}{\lambda} \sqrt{r^{2}-d_{y}^{2}} \sin \alpha_{k}+j \varphi_{M}} \\
\Phi(k, k)=e^{j \frac{2 \pi}{\lambda}\left(d_{y} \cos \alpha_{k}-d_{a} \sin \alpha_{k}+\varphi_{M}-\varphi_{2}\right)}
\end{gathered}
$$

Step4: form simultaneous equations to solve DOA, amplitude and phase errors.

Amplitude errors can be figured out directly from (17), (18).

$$
\begin{gathered}
G_{2}=\operatorname{abs}\left(a_{2 k}\right) \\
G_{M}=\operatorname{abs}\left(a_{M k}\right)
\end{gathered}
$$

As to phase errors, from (17), (18) and (19) we can obtain:

$$
\begin{aligned}
& \operatorname{angle}\left(a_{2 k}\right)=\frac{2 \pi}{\lambda} d_{x} \sin \alpha_{k}+\varphi_{2} \\
& \operatorname{angle}\left(a_{M k}\right)=\frac{2 \pi}{\lambda}\left(d_{y} \cos \alpha_{k}+\sqrt{r^{2}-d_{y}^{2}} \sin \alpha_{k}\right)+\varphi_{M} \\
& \operatorname{angle}(\Phi(k, k))=\frac{2 \pi}{\lambda}\left(d_{y} \cos \alpha_{k}-d_{a} \sin \alpha_{k}\right)+\varphi_{M}-\varphi_{2}
\end{aligned}
$$

Since (24) is just a linear combination of (23) and (24), there are actually only two equations about 
three unknowns $\alpha_{k}, \varphi_{2}, \varphi_{M}$.But when there are two sources in the received signal, we can get four equations about $\alpha_{1}, \alpha_{2}, \varphi_{2}$ and $\varphi_{M}$.

$$
\begin{aligned}
& \operatorname{angle}\left(a_{21}\right)=\frac{2 \pi}{\lambda} d_{x} \sin \alpha_{1}+\varphi_{2} \\
& \operatorname{angle}\left(a_{M 1}\right)=\frac{2 \pi}{\lambda}\left(d_{y} \cos \alpha_{1}+\sqrt{r^{2}-d_{y}{ }^{2}} \sin \alpha_{1}\right)+\varphi_{M} \\
& \operatorname{angle}\left(a_{22}\right)=\frac{2 \pi}{\lambda} d_{x} \sin \alpha_{2}+\varphi_{2} \\
& \operatorname{angle}\left(a_{M 2}\right)=\frac{2 \pi}{\lambda}\left(d_{y} \cos \alpha_{2}+\sqrt{r^{2}-d_{y}^{2}} \sin \alpha_{2}\right)+\varphi_{M}
\end{aligned}
$$

The DOA of arrival and phase errors can be obtained through solving the equations. If there is only one source in the received signal, four equations like (25) (28) can also be constructed by combining this group of signal with another group of received signal. Thus the DOA and phase errors can be figured out.

From the previous demonstration, it can be concluded that DOA, amplitude and phase errors can be estimated simultaneously by redundancy generated by applying Spatial Time DOA Matrix Method to one dimension DOA estimation.

\section{Computer Simulation}

Assuming there are two arrays, one is a right triangle whose $d_{x}=13.3 \mathrm{~m}$ and $r=d_{y}=8 \mathrm{~m}$;

the other is a cute one whose $d_{x}=13.3 m, d_{y}=8 m$ and $r=15.52 m$. Simulated sea echo arrive at the arrays from arbitrary direction within $-90^{\circ} \sim 90^{\circ}$. Set the amplitude and phase errors of $x_{2}$ and $x_{M}$ relative to $x_{M}$ as $G_{2}=1.2, G_{M}=0.8, \varphi_{2}=80^{\circ}, \varphi_{M}=110^{\circ}$. Repeat the experiment 20 times respectively when the signal to noise rates (SNR) are $5 \mathrm{~dB}, 10 \mathrm{~dB}$ and $15 \mathrm{~dB}$. Under each SNR, the mean results of the amplitude errors are 1.2 and 0.8 , and the variance of each result is smaller than $10^{-4}$. It verifies the above algorithm can estimate amplitude errors precisely. The results of phase errors estimation are in the following table.

\begin{tabular}{|c|c|c|c|c|c|}
\hline \multirow{2}{*}{$\begin{array}{c}\text { Array } \\
\text { Manifold }\end{array}$} & \multirow{2}{*}{ SNR } & \multicolumn{2}{|c|}{$\varphi_{2}$} & \multicolumn{2}{c|}{$\varphi_{M}$} \\
\cline { 3 - 6 } & & Mean & Variance & Mean & Variance \\
\hline \multirow{2}{*}{$\begin{array}{c}\text { The cute } \\
\text { triangle }\end{array}$} & $5 \mathrm{~dB}$ & 81.76 & 245.46 & 110.72 & 22.56 \\
\cline { 2 - 6 } & $10 \mathrm{~dB}$ & 81.57 & 55.30 & 110.45 & 5.80 \\
\cline { 2 - 6 } & $15 \mathrm{~dB}$ & 80.02 & 18.39 & 110.32 & 2.11 \\
\hline \multirow{2}{*}{$\begin{array}{c}\text { The right } \\
\text { triangle }\end{array}$} & $5 \mathrm{~dB}$ & 81.62 & 237.82 & 110.69 & 22.45 \\
\cline { 2 - 6 } & $10 \mathrm{~dB}$ & 81.44 & 56.50 & 110.32 & 5.95 \\
\cline { 2 - 6 } & $15 \mathrm{~dB}$ & 80.36 & 17.35 & 110.12 & 2.05 \\
\hline
\end{tabular}

It can be concluded from the table that when the SNR is low, a single result of the phase errors may be far from the value previously set because of the noise and the error introduced by process of solving the equations. However, the mean of the results is very close to the set value even under 
low SNR conditions.

\section{Measured Data}

Select the data collected at 11:20 on 26 June, 2004 by OSMAR2000 of Wuhan University. The shapes of the two arrays consisting of three whips are a right triangle and an obtuse one (showed in Fig.2).

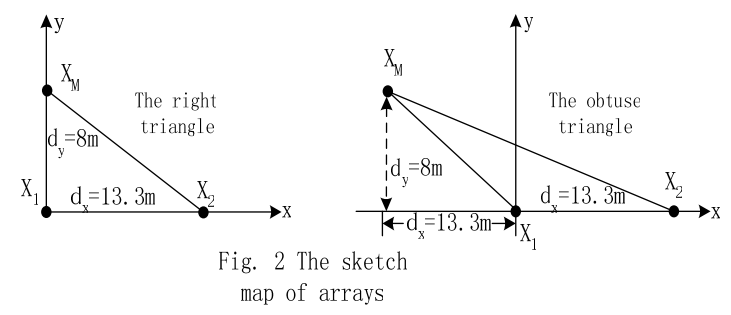

Estimate the DOA of the boat's echo by MUSIC. The following table compares treatment results of measured data before and after calibration with the boat's azimuth measured by GPS.

\begin{tabular}{|c|c|c|c|}
\hline $\begin{array}{c}\text { Array } \\
\text { Manifold }\end{array}$ & GPS Data & $\begin{array}{c}\text { Result } \\
\text { before } \\
\text { calibration }\end{array}$ & $\begin{array}{c}\text { Result after } \\
\text { calibration }\end{array}$ \\
\hline $\begin{array}{c}\text { Right } \\
\text { triangle }\end{array}$ & \multirow{2}{*}{$-24.72^{\circ}$} & $-12.62^{\circ}$ & $-24.93^{\circ}$ \\
\cline { 1 - 3 } $\begin{array}{c}\text { Obtuse } \\
\text { triangle }\end{array}$ & & $-13.76^{\circ}$ & $-25.21^{\circ}$ \\
\hline
\end{tabular}

This table verifies that the performance of MUSIC has been improved remarkably.

\section{Conclusion}

It can be concluded both from the computer simulation and measured data that this algorithm can calibrate the HF Ground wave radar system's triangular antenna array well so as to greatly improve the performance of MUSIC. Though the calibrating results of phase errors wave around the true errors when the SNR is low, the mean of the results is very close to the true value. All these verify that this algorithm is effective and valid.

\section{Reference}

[1] Schmidt R O. Multiple Emitter Location and Signal Parameter Estimation[J]. IEEE Trans on Antenna and Propagation, 1986, AP-34(3):276-280

[2] Roy R, Kailath T. ESPRIT-estimation of signal parameters via rotational invariance techniques. IEEE trans on ASSP, 1986, 37(7):984-995

[3] Weiss A J, Friedlander B. Effects of modeling errors on the resolution threshold of Music algorithm[J]. IEEE Trans on Signal Processing 1994,42(6) : 1519-1526.

[4] Li F, Vaccaro R J. Sensitivity analysis of DOA estimation algorithms[J]. IEEE Trans on Aerospace and Electronic Systems, 1992, 28(3):708-717.

[5] Friedlander B. A sensitivity analysis of the Music algorithm[J]. IEEE Trans on ASSP, 1990, 38(10) : 1740-1751.

[6] Weiss A J, Friedlander B. Eigenstructure methods for direction finding with sensor gain and phase uncertainties [J]. Circuits, Systems, and Signal Processing, 1990, 9(3) : 271-300.

[7] Viberg M, Swindlehurst A L. A Bayesian approach to auto-calibration for parametric array 
signal processing[J]. IEEE Trans on Signal Processing, 1994, 42(12) : 3495-3507.

[8] Hung E. A critical study of a self-calibration direction finding method for arrays[J]. IEEE Trans on Signal Processing, 1994, 42(2) : 471-474.

[9] Jin Liang, Yin Qinye. Space -Time DOA Method. Acta Electronica Sinica, 2000, 28(6) : 8-12.

[10] Kenneth E Laws, Daniel M Fernandez. Simulation-Based evaluations of HF Radar ocean current algorithms[J]. IEEE J Ocean Engr, 2000,25(4) : 481-491. 